Copyright (C) 2006 IEEE. Reprinted from

IEEE Journal of Selected Topics in Quantum Electronics, 2006; 12

(3):360-370

This material is posted here with permission of the IEEE. Such permission of the IEEE does not in any way imply IEEE endorsement of any of the University of Adelaide's products or services. Internal or personal use of this material is permitted. However, permission to reprint/republish this material for advertising or promotional purposes or for creating new collective works for resale or redistribution must be obtained from the IEEE by writing to pubs-permissions@ieee.org.

By choosing to view this document, you agree to all provisions of the copyright laws protecting it. 


\title{
Self-Phase Modulation-Based Integrated Optical Regeneration in Chalcogenide Waveguides
}

\author{
Vahid G. Ta'eed, Student Member, IEEE, Mehrdad Shokooh-Saremi, Libin Fu, Ian C. M. Littler, \\ David J. Moss, Member, IEEE, Martin Rochette, Benjamin J. Eggleton, Yinlan Ruan, \\ and Barry Luther-Davies, Senior Member, IEEE
}

\begin{abstract}
We demonstrate integrated all-optical $2 \mathrm{R}$ regenerators based on Kerr optical nonlinearities (subpicosecond response) in chalcogenide glass waveguides with integrated Bragg grating filters. By combining a low loss $\mathrm{As}_{2} \mathrm{~S}_{3}$ rib waveguide with an inwaveguide photo-written Bragg grating filter, we realize an integrated all-optical $2 \mathrm{R}$ signal regenerator with the potential to process bit rates in excess of $1 \mathrm{~Tb} / \mathrm{s}$. The device operates using a combination of self phase modulation induced spectral broadening followed by a linear filter offset from the input center wavelength. A nonlinear power transfer curve is demonstrated using $1.4 \mathrm{ps}$ pulses, sufficient for suppressing noise in an amplified transmission link. We investigate the role of dispersion on the device transfer characteristics, and discuss future avenues to realizing a device capable of operation at subwatt peak power levels.
\end{abstract}

Index Terms-Chalcogenide glass, gratings, integrated optics, nonlinear optics, optical planar waveguides, optical propagation in nonlinear media, optical signal processing, self-phase modulation.

\section{INTRODUCTION}

O PTICAL DATA transmission rates have recently reached the stage where laboratory demonstrations at $640 \mathrm{~Gb} / \mathrm{s}$ have been reported [1]. At these bit rates, signal processing and reconditioning needs to be done all-optically, since electronic processing speeds are currently limited to around $40 \mathrm{~Gb} / \mathrm{s}$. While all-optical devices such as signal regenerators, wavelength converters, and switches capable of operating at these speeds have been reported, they rely on long lengths of optical fiber and are far from representing practical, compact solutions [2]-[4]. To date, integrated all-optical devices have been based on semiconductor optical amplifiers [5] which are limited in speed due to carrier dynamics. The aim of integrated all-optical devices as-

Manuscript received September 1, 2005; revised February 10, 2006. This work was supported by the Australian Research Council (ARC). CUDOS (the Centre for Ultrahigh-bandwidth Devices for Optical Systems) is an ARC Centre of Excellence.

V. G. Ta'eed, L. Fu, I. C. M. Littler, D. J. Moss, M. Rochette, and B. J. Eggleton are with the Centre for Ultrahigh-bandwidth Devices for Optical Systems (CUDOS), School of Physics, University of Sydney, Sydney, NSW 2006, Australia (e-mail: vahid@physics.usyd.edu.au; libin@physics.usyd.edu.au; littler@physics.usyd.edu.au; dmoss@physics.usyd.edu.au; martinrochette2002 @yahoo.ca; egg@physics.usyd.edu.au).

M. Shokooh-Saremi is with the Centre for Ultrahigh-bandwidth Devices for Optical Systems (CUDOS), School of Physics, University of Sydney, Sydney, NSW 2006, Australia, and also with the Faculty of Electrical Engineering and Robotics, Shahrood University of Technology, Shahrood, Iran (e-mail: mehrdad@physics.usyd.edu.au).

Y. Ruan and B. Luther-Davies are with the Centre for Ultrahigh-bandwidth Devices for Optical Systems (CUDOS), Laser Physics Centre, The Australian National University, Canberra, ACT 0200, Australia (e-mail: yinlan.ruan@ adelaide.edu.au; bld111@rsphymail.anu.edu.au).

Digital Object Identifier 10.1109/JSTQE.2006.872727 sumes operation based on a third-order nonlinearity (e.g., Kerr), and has not yet been realized.

Chalcogenide glasses $(\mathrm{ChG})$ have attracted significant attention in recent years as a promising nonlinear material for alloptical devices [6]-[8]. They are amorphous materials containing at least one of the nonoxide group VI element $(\mathrm{S}, \mathrm{Se}$, and Te)-a chalcogen - combined with other elements such as Ge, $\mathrm{As}, \mathrm{Sb}$, and Ga [8], [9]. Some of the properties that have made them attractive for all-optical devices include the following. 1) Transparency in the near-infrared (IR) as well as into the farIR due to the relatively high atomic masses and weak bond strengths resulting in low phonon energies [8], [10]. 2) Photosensitivity to band gap light. The weak bonding arrangements and inherent structural flexibility result in many distinct photoinduced phenomena [8]. Photodarkening and photobleaching, which shift the absorption edge and induce changes in the refractive index [8], are particularly noteworthy and have been used to write both channel waveguides [9], [11]-[15] and periodic structures [16]-[22]. 3) High refractive index $(\mathrm{n} \approx 2-3)$ providing strong mode field confinement [23], tight bending radii, and allowing complete band gaps to be engineered in photonic crystal structures [24]. 4) Large third-order nonlinearities, with reported values for the nonlinear refractive index $\left(n_{2}\right)$ up to three orders of magnitude larger than silica, and even more for the Raman gain coefficient $\left(g_{R}\right)$ [6], [8], [25]. Since these nonlinearities are based on nonresonant virtual electronic transitions, the intrinsic response time is in the tens of femtoseconds, making devices based on these materials potentially much faster than those based on real carrier dynamics such as semiconductor optical amplifiers [25], [6]. 5) Low two-photon absorption, due to the narrow band gap of these glasses [6]. This is of particular importance as figures of merit $\left(\mathrm{FOM}=n_{2} / \beta \lambda\right.$, in which $\beta$ is two-photon absorption coefficient) greater than unity are required for efficient all-optical devices [26].

While much progress has been made in investigating the basic properties of $\mathrm{ChG}$ as well as the nonlinear properties of $\mathrm{ChG}$ fiber, integrated all-optical signal processing $\mathrm{ChG}$ devices have yet to be demonstrated. In this paper, we demonstrate an integrated, all-optical signal regenerator capable of operating with subpicosecond pulses, based on Kerr nonlinearities in highly nonlinear chalcogenide glass $\left(\mathrm{As}_{2} \mathrm{~S}_{3}\right)$ waveguides. It employs a principle first proposed by Mamyshev [27], operating via a combination of nonlinear self-phase modulation induced spectral broadening followed by spectral filtering. It consists of a low loss chalcogenide glass rib waveguide followed by a high quality integrated waveguide Bragg grating written with a novel 

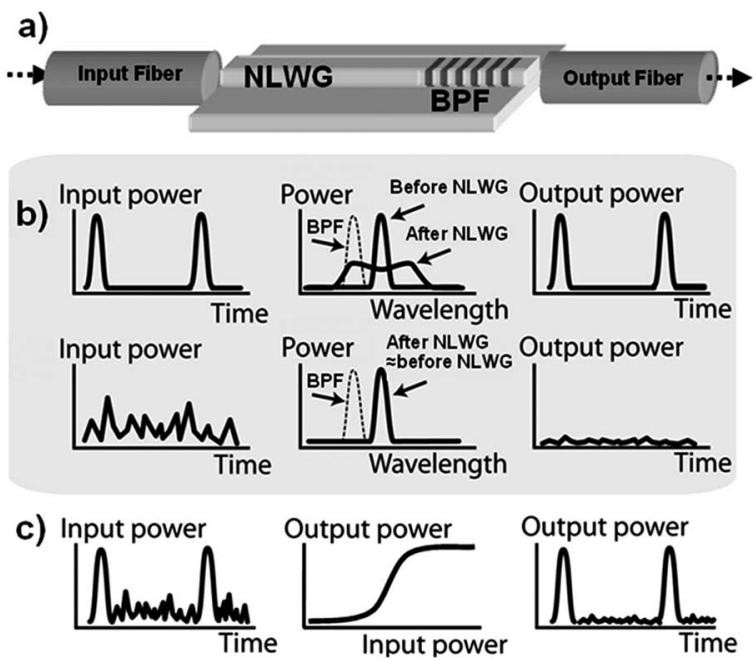

Fig. 1. (a) Physical device architecture. (b) Noise experiences less SPM spectral broadening in the nonlinear waveguide (NLWG) than signal, and hence is attenuated more after the off-center bandpass filter (BPF). (c) Nonlinear power transfer curve which can be used to suppress noise.

Sagnac interferometer [28]. We investigate devices based both on single reflection Bragg gratings as well as multigrating bandpass filters. We characterize the regenerator using $1.4 \mathrm{ps}$ optical pulses with peak optical powers of approximately $50 \mathrm{~W}$, yielding a nonlinear power transfer function. Such transfer functions can be used for optical signal-to-noise ratio (OSNR) and bit-error ratio (BER) improvement [29]. We show that the incorporation of a band pass filter significantly improves the power transfer function over a device with a single reflection band Bragg grating. Finally, we discuss future roadmaps in order to realize devices operating at subwatt peak powers.

The remainder of this paper is structured as follows. In Section II, we present the principle of the device operation and overall design. In Section III, we discuss key optical properties of $\mathrm{As}_{2} \mathrm{~S}_{3}$ and device fabrication processes. We briefly review the method used for fabricating low-loss rib waveguides by ultrafast pulse laser deposition, as well as generating strong Bragg gratings using the inherent photosensitivity of $\mathrm{As}_{2} \mathrm{~S}_{3}$ to visible light through a modified Sagnac interferometric writing setup. In Section IV, we consider nonlinear pulse propagation in these waveguides and present experimental results for the effect of the Kerr nonlinearity in generating new frequency components via SPM, and note the absence of any limiting nonlinear absorption effects. We briefly review the standard description of SPM as lumped nonlinearity, and find good agreement when comparing experimental results to theory. In Section V, we demonstrate our integrated regenerator device concept in two different architectures and analyze device performance, focusing in particular on filter design. In Section VI, we discuss methods to improve the regenerator performance and future devices based on the $\mathrm{ChG}$ waveguide incorporating a Bragg grating. Finally, we present our conclusions in Section VII.

\section{Principle of Device Operation}

Fig. 1 illustrates the principle of device operation. A section of straight, low loss, ChG rib waveguide is followed by an inte- grated Bragg grating filter-either a single grating for operation in reflection, or a double grating forming a band pass filter. The grating center wavelength is offset from the signal wavelength by more than the signal optical bandwidth so that at low intensities, the optical signal is blocked by the filter. At high intensities, (representing logical " $1 \mathrm{~s}$ ") the pulses undergo sufficient spectral broadening through self phase modulation so that a portion is transmitted through the pass band. At still higher intensities, the signal experiences significant broadening and the power within the transmission band pass saturates. The resulting "S-shaped" nonlinear power transfer curve is a hallmark of optical regenerators (for reshaping), and has the effect of reducing noise on both the signal " 0 's" and " 1 's," improving the optical signal to noise ratio (OSNR). Further, it has recently been shown that these regenerators attenuate spontaneous emission noise more than a pulsed signal, resulting in a direct BER improvement [30], [31].

Typically, such regenerators have relied on either large spools of silica fiber, or meter lengths of highly nonlinear glass fiber to generate the necessary nonlinear phase shift. Here, we demonstrate an integrated regenerator device using a $5 \mathrm{~cm}$ long, highly confining, nonlinear $\mathrm{ChG}$ rib waveguide to generate a nonlinear phase change of $\phi_{\mathrm{NL}}=3 \pi / 2$, while the filtering is accomplished with an integrated holographically written Bragg grating offset from the carrier wavelength. We experimentally demonstrate our device using 1.4-ps pulses, obtaining a nonlinear transfer curve suitable for suppressing noise. As previously mentioned, the potential speed of this device is subpicosecond since it is based on a nonresonant Kerr nonlinearity.

\section{WAVEGUIDE AND GRATING FABRICATION}

Our devices are based on $\mathrm{As}_{2} \mathrm{~S}_{3} \mathrm{ChG}$, which has recently formed the basis of low-loss rib waveguides [23]. While not exhibiting the largest $n_{2}$ of all ChGs, $\mathrm{As}_{2} \mathrm{~S}_{3}$ still displays an $n_{2}$ of $\sim 100 \times$ silica $\left(3.8 \times 10^{-18} \mathrm{~m}^{2} / \mathrm{W}\right.$ at $\left.1.55 \mu \mathrm{m}\right)$, and has been studied carefully. The band gap energy of $\mathrm{As}_{2} \mathrm{~S}_{3}$ is $2.4 \mathrm{eV}$ $\left(\lambda_{\text {gap }}=517 \mathrm{~nm}\right)$, and its linear $\left(n_{0}\right)$ refractive index is 2.38 . Furthermore, a nonlinear FOM of 12 has been measured in $\mathrm{As}_{2} \mathrm{~S}_{3}$ in the telecommunication wavelength band $(1.55 \mu \mathrm{m})$. In addition, $\mathrm{As}_{2} \mathrm{~S}_{3}$ is photosensitive to visible light, and grating structures may be written using $\mathrm{Ar}^{+}(514 \mathrm{~nm})$, doubled Nd:YAG $(532 \mathrm{~nm})$, and $\mathrm{He}-\mathrm{Ne}(632.8 \mathrm{~nm})$ lasers via photodarkening.

In this section, we discuss the film deposition by ultrafast pulse laser deposition and fabrication of rib waveguides by dry etching, and then describe our recent work in writing apodized Bragg gratings using a modified Sagnac interferometer.

\section{A. Deposition and Fabrication of $A s_{2} s_{3}$ Rib Waveguides}

Methods for fabricating thin films of ChG include thermal evaporation, sputtering, spin deposition, and pulsed laser deposition. We prepared our samples using ultrafast pulsed laser deposition (UFPLD), which produces high quality films that accurately reproduce the stoiciometry of the target [12]. $\mathrm{As}_{2} \mathrm{~S}_{3}$ layers, typically of thickness $2.4 \mu \mathrm{m}$, were deposited onto a $\mathrm{SiO}_{2} / \mathrm{Si}$ substrate employing a frequency doubled, mode locked $\mathrm{Nd}$ :YAG laser. The thickness of the thermally grown $\mathrm{SiO}_{2}$ layer is $\sim 2.4 \mu \mathrm{m}$ and provides a substrate with refractive index less 


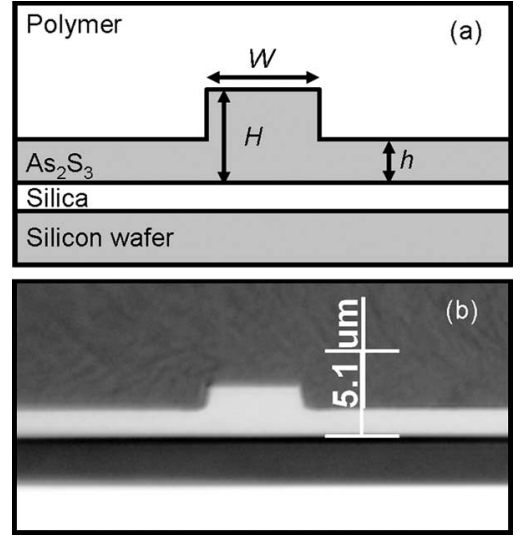

Fig. 2. (a) Schematic cross section of an As2S3-based rib waveguide. (b) An optical micrograph of a waveguide.

than $\mathrm{As}_{2} \mathrm{~S}_{3}$. The ChG layers deposited by UFPLD are very dense, high quality, and usually do not need to be annealed before waveguide fabrication [23].

We use a standard photolithographic technique with an aluminum mask followed by dry etching to fabricate the rib waveguides. Etching is based on a Helicon plasma dry etching process with $\mathrm{CF}_{4} / \mathrm{O}_{2}$ gas as the etchant, with an etch rate of 250 $\mathrm{nm} / \mathrm{min}$. After etching the waveguide, a high quality polysiloxane layer $(n=1.53 @ 1.55 \mu \mathrm{m})$ is deposited on the top of the waveguide (thickness $\approx 10-20 \mu \mathrm{m}$ ) to act as an over-cladding and protective layer [23]. Fig. 2 shows a cross section of a typical waveguide. Although these waveguides meet generalized requirements for single mode operation [32], they do support leaky higher order modes [33].

We found that propagation losses, as well as polarizationdependent loss (PDL), for these waveguides varied noticeably based on their cross-sectional dimensions, film growth conditions, and subsequent fabrication processes. Using free space bulk optics, we verified that TE polarized light showed lowest insertion loss and, consequently, all our experimental results are based on TE polarized light. Using both cut-back methods and techniques based on Fabry-Perot waveguide cavity fringe analysis, we confirmed that typical propagation losses are in the region of $0.25 \mathrm{~dB} / \mathrm{cm}$ at $1550 \mathrm{~nm}$.

\section{B. Bragg Gratings Written by Sagnac Interferometer}

We use a holographic grating writing setup based on a modified Sagnac interferometer (Fig. 3) for writing Bragg gratings in the $\mathrm{As}_{2} \mathrm{~S}_{3}$ rib waveguides [28]. In reports to date of holographically written Bragg gratings in ChG-based fibers and waveguides, Mach-Zehnder (MZ) interferometers have been employed [17], [18], [20]-[22], which are very sensitive to environmental perturbations (mechanical vibrations and component lag, thermal gradient induced strain, as well as air currents). This affects the stability of the interference pattern over time and degrades the quality of the Bragg grating - particularly for gratings requiring long writing times. In contrast, a Sagnac interferometer provides excellent stability over time and, with appropriate modifications, the waveguide can be incorporated

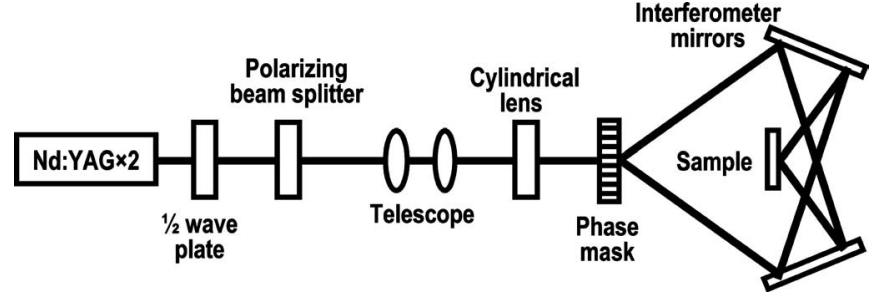

Fig. 3. Schematic of the grating writing setup based on the modified Sagnac interferometer.

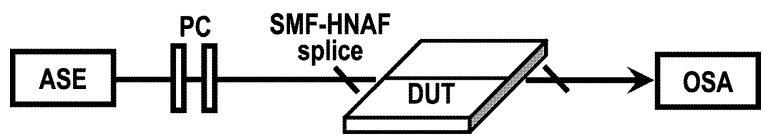

Fig. 4. Grating characterization setup. ASE: amplified spontaneous emission. PC: bulk optics polarizer and $1 / 4$ wave plate. SMF: single-mode fiber. HNAF: high numerical aperture fiber. OSA: optical spectrum analyzer.

in the writing setup in a straightforward fashion. Using a vertical vacuum sample holder enabled us to implement the writing system horizontally. Bragg gratings at arbitrary wavelengths could be written by changing the sample holder position and tuning the mirror angles. We used the modified Sagnac interferometer in combination with a $\mathrm{CW}$, frequency-doubled, diode-pumped $\mathrm{Nd}$ :YAG laser $(\lambda=532 \mathrm{~nm})$ with a maximum available power at the sample of $50 \mathrm{~mW}$. The polarized laser beam was telescopically expanded, cylindrically focused, and split using a phase mask with rectangular grooves $\left(\Lambda_{\mathrm{m}}=1063.3 \mathrm{~nm}\right)$. The laser had a short coherence length $(\sim 3 \mathrm{~mm})$ in order to shape the grating profiles via the length-dependent visibility of the interference pattern [34], resulting in a nearly ideal raised Gaussianapodized grating $\sim 3 \mathrm{~mm}$ in length.

Fig. 4 shows a schematic view of the setup used to characterize the Bragg gratings using an unpolarized high-power EDFAASE source (C-band: 1520-1580 nm), and an optical spectrum analyzer (OSA) with 60-pm resolution. The output of the source was butt-coupled into the waveguide via a high-NA fiber. A second high-NA fiber coupled the transmitted output either to the detector for alignment optimization or to the OSA to measure the transmission spectra. A bulk optics polarizer and quarter wave plate was placed between the sample and light source, and adjusted to obtain the maximum polarization extinction ratio for the two orthogonal polarization states.

Fig. 5 shows the transmission spectra of a strong, high quality Bragg grating for TE polarized light, written in a $4 \mu \mathrm{m}$ wide, $5 \mathrm{~cm}$ long $(H=2.39 \mu \mathrm{m}, h=1.39 \mu \mathrm{m}) \mathrm{As}_{2} \mathrm{~S}_{3}$ rib waveguide using the modified Sagnac setup. The total writing power was $6.0 \mathrm{~mW}$ and the exposure time was $60 \mathrm{~s}$. Both the high quality (sharp spectral features) and strength of the gratings, in terms of spectral width $(\Delta \lambda \sim 5 \mathrm{~nm}$ corresponding to $\Delta n_{\mathrm{ac}} \sim 7.7 \times 10^{-3}$ ) and depth, are indications of the stability of the Sagnac setup. Note that the bottom of the transmission dip is less than $-33 \mathrm{~dB}$, but cannot be resolved due to transmission from alternative higher order mode paths. One of the main features observed in Fig. 5 is the suppression of 


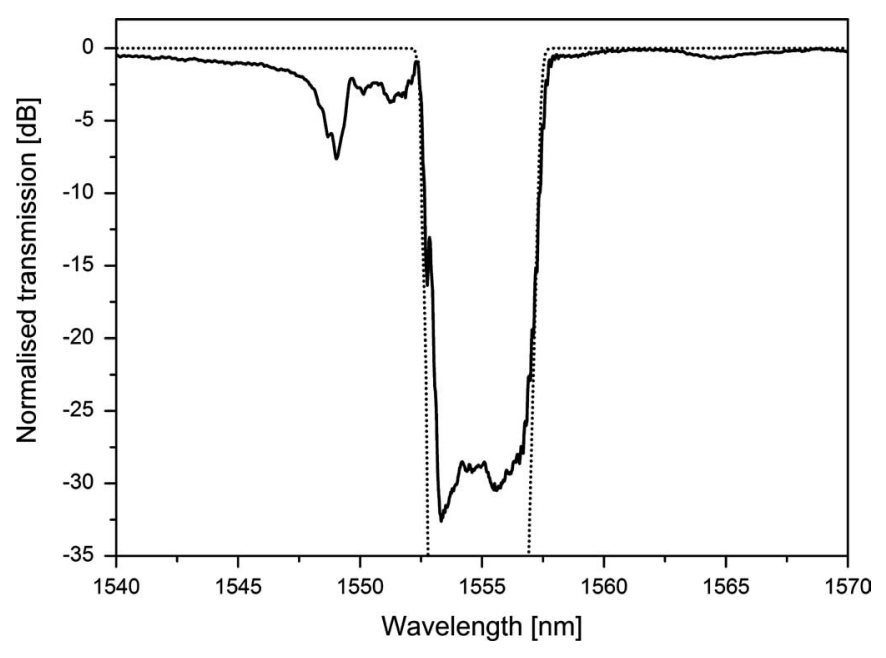

Fig. 5. Normalized transmission spectrum of a strong, well-apodized grating for TE polarization. This grating has been written in a $4-\mu \mathrm{m}$-wide and 5 -cm-long waveguide $(H=2.39 \mu \mathrm{m}, h=1.39 \mu \mathrm{m})$.

side lobes around the main transmission dip. The appearance of side lobes is a result of a Fabry-Perot cavity effect caused by the sharp boundaries of the Bragg grating. The near absence of side lobes in our transmission spectra indicates that the grating profile has been suitably apodised through the short coherence of the writing laser.

As we mentioned, the waveguides used for the experiments supported multiple leaky modes. Weak transmission dips (such as the shallow transmission dip near $1545 \mathrm{~nm}$ ) related to these higher order modes (HOMs) are present in the transmission spectra of the gratings. Due to the lower overlap between these modes and the fundamental mode, the reflectivity is less, and the resulting grating spectrum is weaker [33].

To model the grating spectral response, we used a method based on $2 \times 2$ transfer matrices for thin film structures. The entire grating structure was discretized into $m$ layers, each of thickness equal to a quarter of the grating period $(\Lambda)$. Each layer is then described by a matrix which includes the effects of reflection, transmission, and the phase changes induced by the layer. The complex reflectivity and transmission of the entire grating for a single wavelength is then given by the $m$ multiplied matrices. This method is particularly flexible and can model arbitrarily complex gratings, limited only by the number of layers that are modeled. Fig. 5 also shows that the theoretical fit using this method agrees well with the experimentally measured grating transmission spectra.

\section{Nonlinear Pulse Propagation}

In this section, we examine the nonlinear properties of our waveguides, presenting experimental results for the measured nonlinear absorption and spectral broadening due to self phase modulation for intense pulses propagating through $5 \mathrm{~cm}$ of bare (no grating) waveguide, followed by a theoretical discussion.

\section{A. Experimental Setup and Results}

Fig. 6 shows the experimental setup used in these experiments. Transform-limited, hyperbolic secant squared $\left(\mathrm{sech}^{2}\right)$

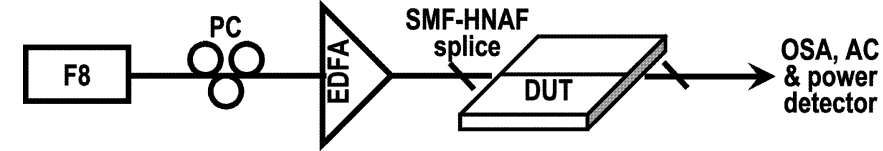

Fig. 6. Generalized schematic layout for experiment to measure self-phase modulation, nonlinear absorption, and pulse regeneration in a chalcogenide rib waveguide. F8: figure-8 laser. PC: polarization controller. EDFA: erbium doped fiber amplifier. SMF: single mode fiber. HNAF: high numerical aperture fiber. DUT: rib waveguide device under test. OSA: optical spectrum analyzer. AC: pulse autocorrelator.

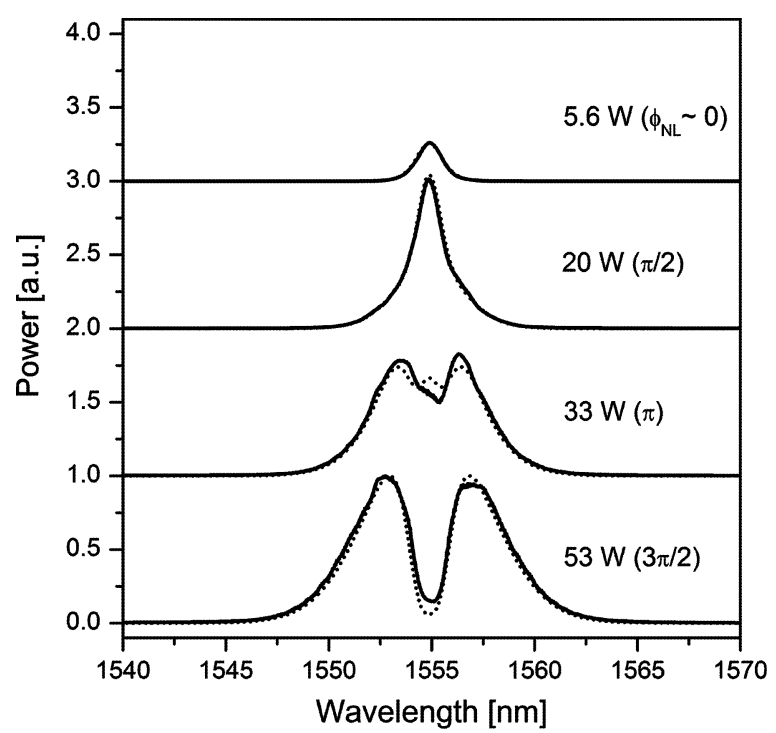

Fig. 7. Symmetric spectral broadening due to self-phase modulation with increasing peak power within a $5-\mathrm{cm}$ chalcogenide rib waveguide. Solid line is experiment and dotted line is theory. The pulse peak power calculated within the waveguide, and the nonlinear phase shifts $(\phi N L)$ are estimated from the experimental spectral shape.

shaped pulses were generated from a custom-built, mode-locked "figure-8" laser [36]. Pulse duration (FWHM) was tunable from 1.3 to $2.5 \mathrm{ps}$, while the lasing wavelength could be changed from 1538 through to $1556 \mathrm{~nm}$. The repetition rate was set at $3.58 \mathrm{MHz}$. The output of the laser was passed through a polarization controller and then a custom optical amplifier designed to minimize SPM within the amplifier itself, resulting in nearly transform limited pulses, with a 1.48-nm 3-dB spectral width and 1.8-ps pulsewidth at $1554.9 \mathrm{~nm}$.

The output of the amplifier was coupled through a short $(<20 \mathrm{~cm})$ length of SMF spliced to a short $(5 \mathrm{~mm})$ length of high NA fiber, and then butt-coupled into the waveguide. The purpose of the high NA fiber was to improve mode matching between the SMF and waveguide. The total insertion loss (with high NA fiber) of the waveguide, fiber to fiber, was $\sim 9.3$ $\mathrm{dB}$, of which about $1.5 \mathrm{~dB}$ was propagation loss, equating to a coupling efficiency of $\sim 3.9 \mathrm{~dB}$ per facet, of which $0.8 \mathrm{~dB}$ was Fresnel loss. The output of the waveguide was directed either into a power meter, optical spectrum analyzer, or pulse autocorrelator.

Fig. 7 shows the transmitted pulse spectra after passing through the bare waveguide showing significant broadening with increasing input peak power, due to SPM. The maximum 


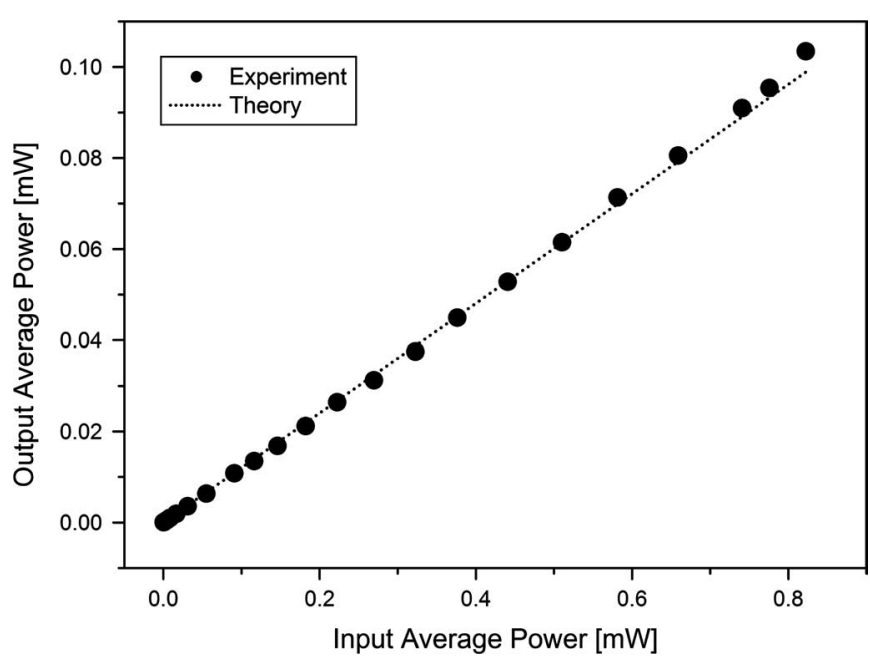

Fig. 8. Output average power as a function of input average power showing no nonlinear absorption. The maximum measured input average power of 0.82 $\mathrm{mW}$ is equal to a calculated peak power within the waveguide of $53 \mathrm{~W}$, and corresponds to an intensity of $1.0 \mathrm{GW} / \mathrm{cm}^{2}$.

observed broadening resulted in two peaks, corresponding to nonlinear optical phase shift $\phi_{\mathrm{NL}} \approx 3 \pi / 2$. The excellent symmetry of the spectral broadening is a result of the nearly ideal transform limited pulses. The maximum peak power in the waveguide was $53 \mathrm{~W}$, corresponding to an intensity of 1.0 $\mathrm{GW} / \mathrm{cm}^{2}$. We also measured the pulse spectra after the amplifier and directly before the device to verify that SPM induced spectral broadening by the amplifier and pigtails was negligible.

Fig. 8 shows the experimental power transfer function for a waveguide with no grating present, exhibiting a linear relation, indicative of low nonlinear (two-photon) absorption, in agreement with the FOM we [23] and others [25] have calculated for $\mathrm{As}_{2} \mathrm{~S}_{3}$. The small random deviations from a linear relationship were attributed to a variation in coupling efficiency due to thermal/mechanical changes that was $<0.5 \mathrm{~dB}$ during the course of experiments. In other experiments where we attempted to measure even greater nonlinear phase shift induced spectral broadening, we observed irreversible waveguide loss. This phenomena has been observed at $1.5-\mu \mathrm{m}$ wavelengths in $\mathrm{As}_{2} \mathrm{~S}_{3}$ at similar threshold values $\left(0.8-1.3 \mathrm{GW} / \mathrm{cm}^{2}\right)$ in thermally evaporated films, but not in bulk or fiber [37]. The high quality of the ultrafast pulsed laser deposited films may contribute to an increased threshold for this effect.

\section{B. Modeling the Waveguide as Lumped Nonlinearity}

In this section, we discuss the physics of nonlinear propagation in these waveguides, including a discussion of the effects of dispersion. We found that the large normal material dispersion in ChG in the $1550 \mathrm{~nm}$ wavelength range can potentially be exploited to "wash out" the oscillations normally present in SPM broadened spectra. This would particularly apply for long device lengths. For our devices, however, since the typical propagation lengths are on the order of a few centimeters, dispersion effects remain small, and a "lumped" element approach is appropriate. Nonetheless, for the future, for longer devices that could potentially operate at much lower peak powers, the large normal dispersion of $\mathrm{ChG}$ remains a potential feature to be exploited to improve device performance over silica or bismuth oxide-based devices.

In third order nonlinear materials such as the $\mathrm{ChG}$, the Kerr nonlinearity results in a nearly instantaneous intensitydependent index of refraction (response time $<50 \mathrm{fs}$ ) represented by $n_{2}$. Over the propagation length $L$, this change in local refractive index generates a nonlinear phase change $\left(\phi_{\mathrm{NL}}\right)$, and so for pulses $(u(z, t))$ propagating through a nonlinear $\mathrm{ChG}$ waveguide, the normalized output field amplitude $u(z, t)$ is

$$
u(z, t)=u(0, t) \exp \left[i \phi_{N L}(z, t)\right]
$$

where the nonlinear phase is

$$
\phi_{N L}(z, t)=|u(0, t)|^{2} L / L_{N L}
$$

Here, $L_{\mathrm{NL}}$ is the characteristic nonlinear length

$$
L_{N L}=\frac{1}{P_{0} \gamma}=\frac{c A_{\mathrm{eff}}}{P_{0} n_{2} \omega_{0}}
$$

where $P_{0}$ is the peak power, $A_{\text {eff }}$ is the effective cross section of waveguide, and $\omega_{0}$ is the carrier frequency. Physically, $L_{\mathrm{NL}}$ defines the length scale over which one radian phase shift is accumulated. Normally in (2), the path length $L$ is replaced by a effective length $L_{\text {eff }}=(1-\exp [-\alpha L]) / \alpha$ in order to account for propagation loss $(\alpha)$. [38]

In pulses of sufficient intensity, this SPM introduces a frequency chirp, with the leading edge of the pulse shifting toward longer wavelengths and the trailing edge shifting toward the shorter wavelengths. Overall, the pulse spectral shape is broadened and shows oscillatory structure. Physically, these oscillations originate due to the interferences of different components of the pulse at the same frequency, but with differing phases. The number of spectral peaks is linearly dependent upon the maximum nonlinear phase change that is generated; however, the actual spectral shape also depends on the dispersive properties of the medium [38].

For waveguides made of $\mathrm{ChG}$, the zero dispersion wavelength tends towards the mid-infrared $\left(5 \mu \mathrm{m}\right.$ for $\left.\mathrm{As}_{2} \mathrm{~S}_{3}\right)$, resulting in large negative (normal) dispersion in the near-infrared region $\left(D \approx-400 \mathrm{ps}[\mathrm{nm} \mathrm{km}]^{-1}\right.$ for $\mathrm{As}_{2} \mathrm{~S}_{3}$ near $\left.1550 \mathrm{~nm}\right)$ [25]. We define a characteristic dispersion length over which a pulse broadens by a factor of $\sqrt{ } 2$

$$
L_{D}=\frac{\tau_{0}^{2}}{\beta_{2}}
$$

where $\tau_{0}$ is the pulse width and $\beta_{2}$ is the group velocity dispersion. For $1.8 \mathrm{ps}$ pulses in $\mathrm{As}_{2} \mathrm{~S}_{3}$ waveguides, $L_{D}=2.0 \mathrm{~m}$. The relative ratio of the two characteristic lengths $N^{2}=L_{D} / L_{\mathrm{NL}}$, describes the main effects due to the combination of dispersion and nonlinearity. Dispersion dominates for $N \ll 1$, and SPM dominates for $N \gg 1$, while for intermediate values, $N \sim 1$, both SPM and dispersion play equally important roles [38]. To correctly simulate the interplay of dispersion and nonlinearity usually requires the nonlinear Schrödinger equation. In the device presented here, $N \sim 15$ (for the highest peak power) 
and the length of the device $(5 \mathrm{~cm})$ is significantly shorter than $L_{D}=2.0 \mathrm{~m}$; therefore, dispersive effects can be neglected. [38]

For propagation lengths close to $L_{D}$, the large normal dispersion present in $\mathrm{ChG}$ in the 1550 -nm region can linearize pulse frequency chirp, thus reducing the interference that causes the oscillatory structure of SPM broadened spectra, resulting in a smoother, flatter spectra. This is advantageous for SPM-based regeneration [27].

We modeled the spectral results for the experiments using the preceding equations. Based on our results, we estimated a value for the Kerr nonlinear coefficient $n_{2}$ of $2.9 \times 10^{-18}$ $\mathrm{m}^{2} / \mathrm{W}$, or roughly $100 \times$ silica, which is similar to values in the literature [23].

\section{OPTICAL REGENERATION}

The high quality of our waveguides and Bragg gratings provides a platform from which all-optical signal processing devices can be developed. The first device that we have focused on thus far has been an optical pulse $2 \mathrm{R}$ regenerator based on spectral filtering of SPM broadened pulses. As this device does not rely on pump/pulse interaction, its operation is relatively simple to implement; however, more complex functionality can also be readily conceived.

In this section, we examine two integrated regenerators based on a ChG waveguide: the first incorporating a band stop filter, fabricated as a single Bragg grating, while the second is based on a band-pass filter consisting of two Bragg gratings. We also compare the resulting measured device behavior to theory.

\section{A. Single Grating, Band-Stop Filter Regenerator}

First, we present experimental results for a device based on a single Bragg grating, forming a band-stop filter. For the results presented here, we used the same grating shown in Fig. 5 (Section II), which had a spectral width of $5 \mathrm{~nm}$ and was physically located $5 \mathrm{~mm}$ from the output end of the waveguide. The input signal wavelength was tuned to within the rejection band of the grating so that at low input powers, the signal was reflected. We investigated cases where the input wavelength was tuned to the center of the Bragg grating stop band, and cases where it was offset slightly by $1.0 \mathrm{~nm}$ (still well within the stop band).

Fig. 9 shows the evolution of the pulse spectra when the laser wavelength was offset $1.0 \mathrm{~nm}$ from the grating central wavelength. At moderate input power, transmission is low since the rejection band of the grating is much wider than the 3-dB spectral bandwidth of the input pulse. As the peak power of the pulses increases, self phase modulation causes the spectral width to increase beyond the spectral width of the grating resonance and, as a result, "leaks" out from either side of the grating. Because the input wavelength was offset by $1.0 \mathrm{~nm}$ from the Bragg grating stop band, however, the "leaked" signal spectrum is asymmetric.

Fig. 10 shows that the device exhibited a clear nonlinear power transfer function, as required for optical regeneration. The two curves are for the input pulse center wavelength centered on the Bragg grating reflection band and offset by $1.0 \mathrm{~nm}$ to longer wavelength, clearly indicating that offsetting the pulse

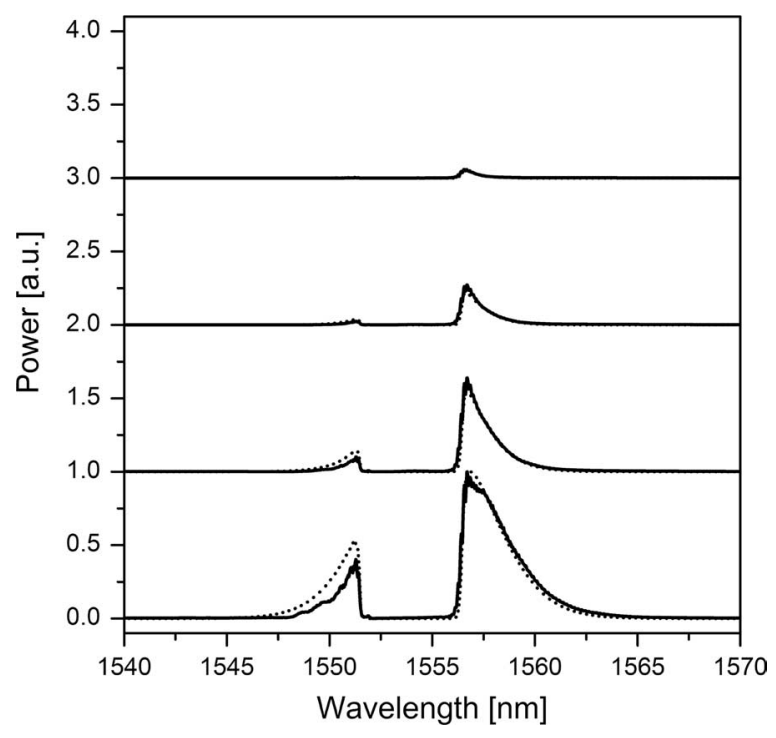

Fig. 9. Spectral evolution for the band-stop device, showing experiment (solid line) and theory (dotted line) for increasing peak powers. The discrepancy seen at the short wavelength side is due to differences between the modeled grating and the actual grating.

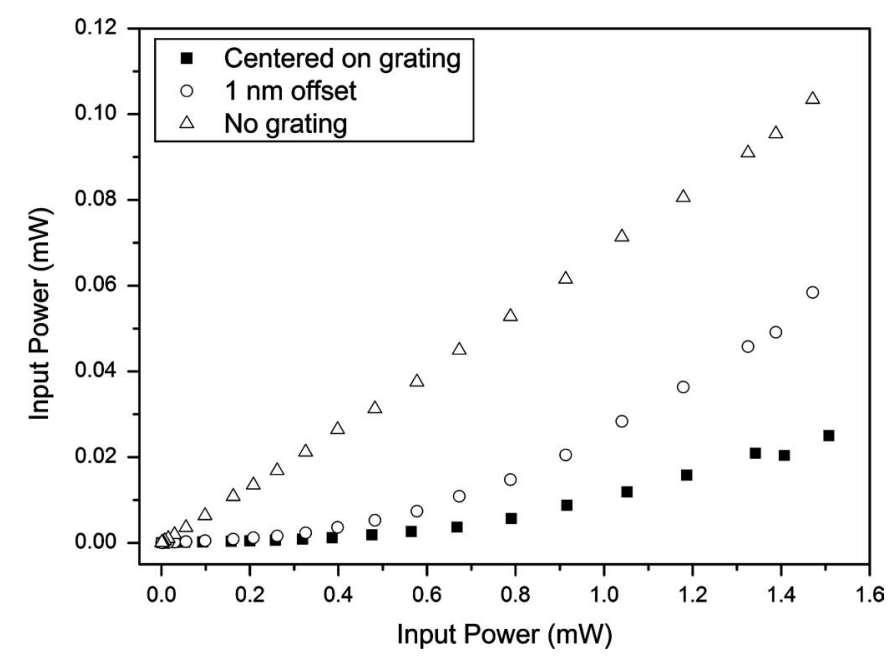

Fig. 10. Band-stop power transfer curves for the input pulse centerd on the grating wavelength and offset $1 \mathrm{~nm}$ to longer wavelength, resulting in a nearly optimal nonlinear transfer curve for this device geometry. The transfer function with no grating present (i.e., only SPM) is shown for reference.

wavelength relative to the Bragg wavelength yields a more optimal nonlinear transfer function.

To model this device, we extended the theory described previously to include spectral filtering through the integrated Bragg grating filter. The output of the device is thus given by

$$
U(z, f)=\Im\left\{u(0, t) \exp \left[i \phi_{N L}(z, t)\right]\right\} \times H(f)
$$

where $\Im$ is the Fourier transform function, $H(f)$ is the complex transmission function of the grating generated using the method described in Section II-B, and $U(z, f)$ is the resulting field amplitude with respect to optical frequency $f$. Theory was compared to experiment in the spectral evolution of Fig. 9, and shows good agreement. 


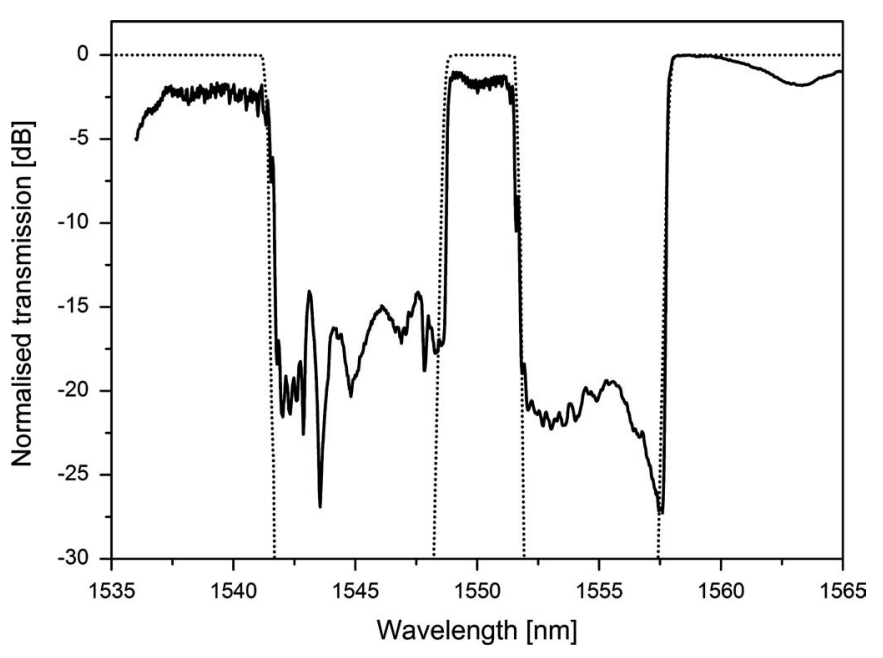

Fig. 11. Experimental (solid) and theoretical (dotted) transmission spectra for the pass-band filter, consisting of two Bragg gratings. The longer wavelength grating was written closest to the output facet of the waveguide. Pass and rejection bands are, respectively, 2.8- and 16.3-nm wide.

Ideally, SPM regenerators would operate with a narrow band pass filter rather than a rejection filter as employed here. This would have the combined effect of controlling the output pulse spectrum as well as introducing a "roll-over" in the power transfer curve at high power, equivalent to optical saturation, or limiting, behavior which would reduce noise on the signal logical " 1 's" as well as the "0's." Next, we turn to results based on these pass-band gratings.

\section{B. Dual Grating, Bandpass Filter Regenerator}

In this section, we present experimental results for an integrated $2 \mathrm{R}$ regenerator based on a waveguide integrated with a transmission band pass filter consisting of a dual Bragg grating filter.

Fig. 11 shows the transmission spectrum of a band-pass filter for TE polarized light, as well as the theoretical fit used for the modeling. The filter consisted of two gratings offset from each other to produce an overall rejection bandwidth of $16.3 \mathrm{~nm}$ with a 2.8-nm-wide passband in the middle.

In this experiment, we tuned the figure- 8 laser such that the pulse width was $1.4 \mathrm{ps}$ and the repetition rate was $8.75 \mathrm{MHz}$. The pulse center wavelength was tuned to within the rejection band of the Bragg grating on the long wavelength side of the pass band. As seen in Fig. 12, this resulted in low transmission at low input power, since the filter rejection bandwidth was much wider than the 3-dB spectral bandwidth of the input pulse, similar to the band-stop regenerator. As the input pulse power was increased, SPM broadened the spectrum so that power was transmitted through the passband of the grating. At still higher powers, the broadened spectrum was filtered by the longer wavelength side of the band-stop filter, resulting in saturation behavior observed in the power transfer function in Fig. 13. As mentioned in Section II, this nonlinear "S-shape" function would be suitable for optical signal regeneration.

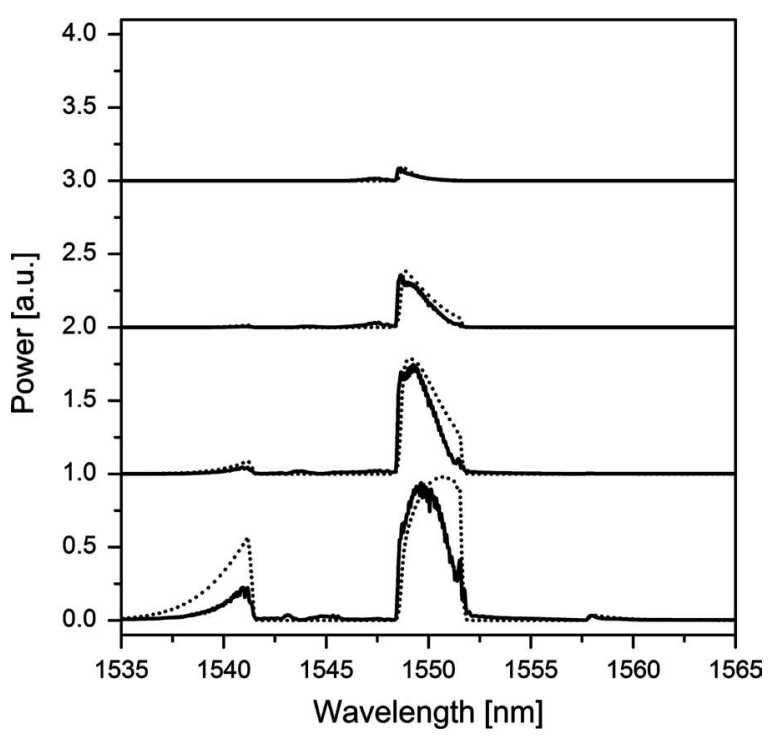

Fig. 12. Transmission spectra for the band-stop device, showing experiment (solid line) and theory (dotted line) for increasing peak powers.

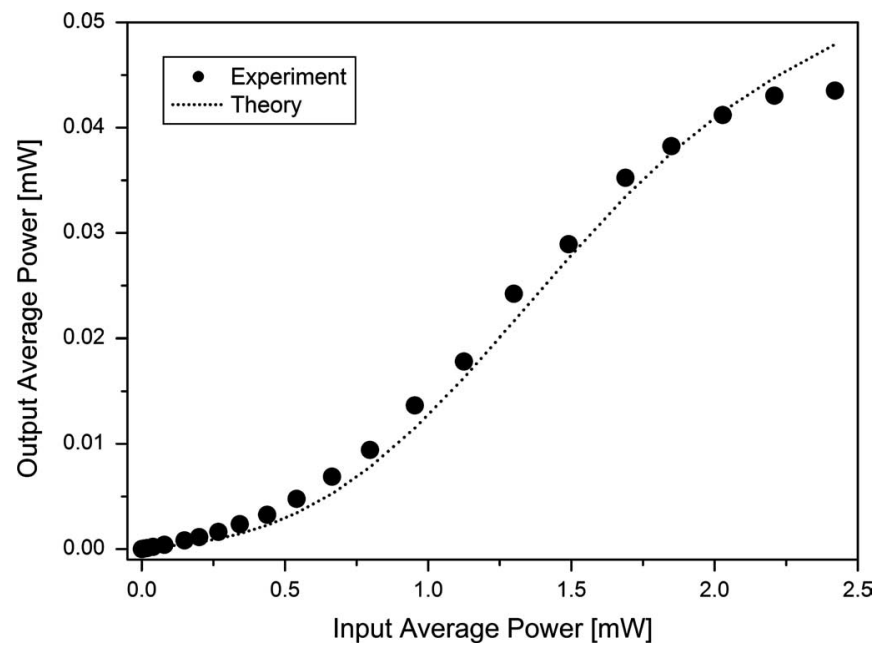

Fig. 13. Output average power as a function of input average power showing characteristic $\mathrm{S}$-shaped nonlinear transfer curve suitable for pulse regeneration and noise suppression.

Maintaining the temporal integrity of the pulses is a key consideration for optical regenerators. The autocorrelation of the input and output pulses at high power is shown in Fig. 14, and indicates that the output pulses were broadened slightly to $\sim 3$ ps. This broadening is not due to either waveguide dispersion or material dispersion in the passive waveguide section (both of which are negligible on this length scale), but rather is due to grating dispersion near the edge of the stop-band [39]. Fig. 15 shows a comparison of the theoretical pulse autocorrelation for this device (b) with and (c) without grating phase effects, and clearly shows narrower pulses when the phase effects are removed. The distorted pulses represent device performance impairment and so the filter design would need to be improved for future devices. Optimizing the grating pass band shape and width is expected to reduce this, and can provide a means for reconfigurable regeneration [40]. 


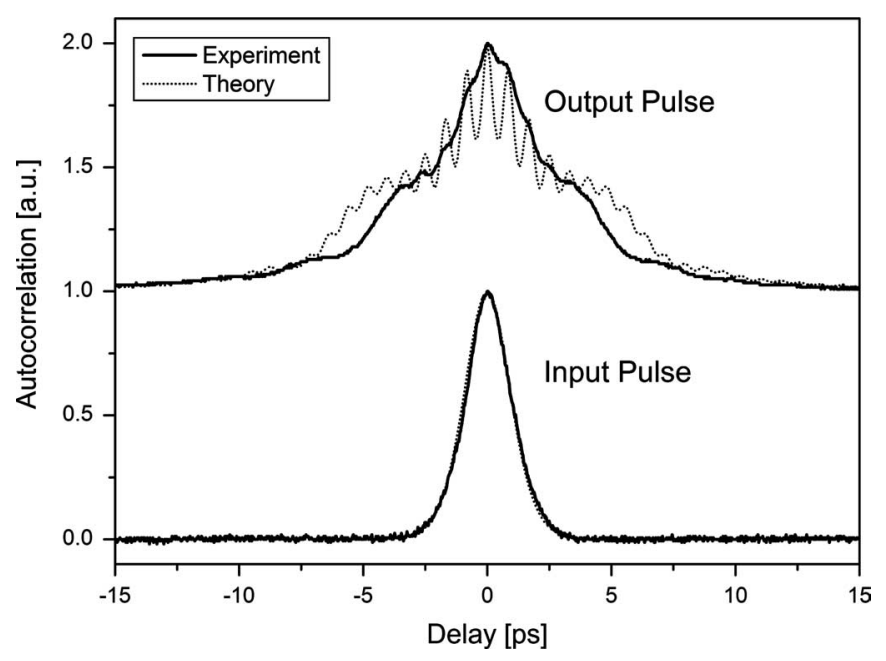

Fig. 14. Experimental and theoretical input/output (offset for clarity) pulse autocorrelation. Pulses broaden from 1.4 to $\sim 3$ ps, mainly due to grating edge dispersion.

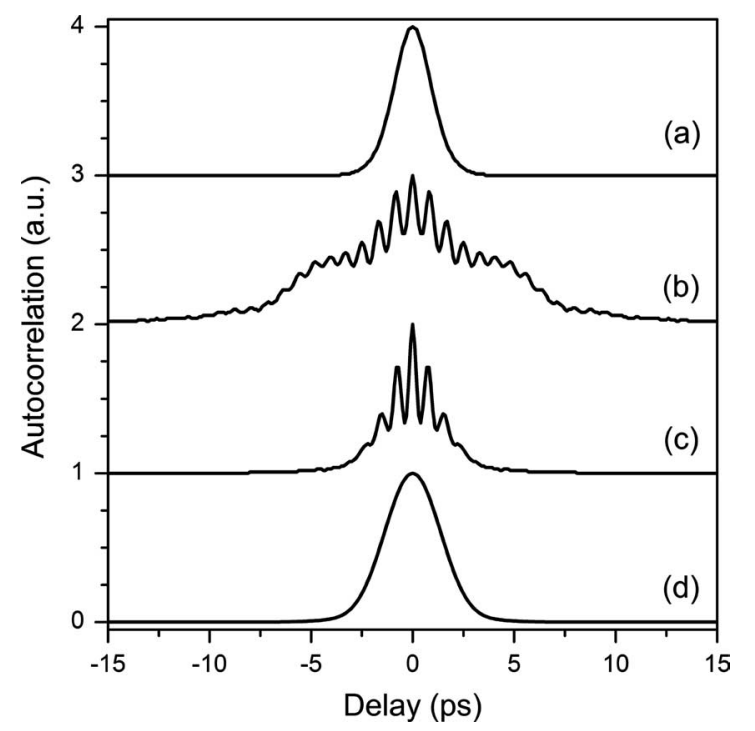

Fig. 15. Theoretical pulse autocorrelation. (a) Input. (b) Output after double grating band pass filter. (c) After the same grating but with phase effects artificially removed. (d) After a weak Gaussian reflection grating based filter.

\section{DISCUSSION}

In this section, we discuss the results and present a roadmap for the improvement of future device performance. The main goals to achieving practical devices center around reducing the required operating peak power, reducing pulse distortion, and improving the nonlinear transfer curve.

The devices demonstrated here operate at peak powers from $10 \mathrm{~W}$ to $50 \mathrm{~W}$, and were tested with low duty cycle optical pulses. For practical operation, the target is to achieve operation with subwatt peak power levels in order to facilitate regeneration of high duty cycle (e.g., 33\% return-to-zero) optical signals. Ultimately, a number of approaches will need to be employed to achieve this goal. First, the optical nonlinearity itself can be increased by a factor of $>4$ by choosing one of the higher nonlinearity glasses which replace the chalcogen $\mathrm{S}$ for Se; e.g., $\mathrm{As}_{2} \mathrm{Se}_{3}$ or $\mathrm{Ge}_{33} \mathrm{As}_{12} \mathrm{Se}_{55}$. While these materials can exhibit greater two photon absorption, this can be advantageously used to flatten the nonlinear transfer function at high peak powers, as has already been achieved with $\mathrm{As}_{2} \mathrm{Se}_{3}$ fiber-based regenerator [41]. Secondly, peak intensities can be increased relative to peak power by reducing mode area. The large $\mathrm{ChG}$ refractive index means that, in principle, waveguide cross sections down to submicrometer dimensions can be achieved. The challenges in this regard center around reducing sidewall roughness in order to shrink the waveguide dimensions to $1 \mu \mathrm{m}^{2}$ while maintaining low propagation loss, as well as to achieve high coupling efficiencies to fiber. Third, operating peak powers can be reduced by increasing the interaction length. Our devices were limited to less than $6 \mathrm{~cm}$ due to our photo mask design and wafer size-not by intrinsic propagation losses. In principle, it is possible to fabricate 10-30 cm waveguides, compacted either as serpentine structures or spirals. Combining all of these approaches, a reduction in operating power by two orders of magnitude is achievable, resulting in subwatt power level operation.

In terms of improving the nonlinear power transfer characteristics of the device, there are a number of approaches. Fabricating longer waveguides would not only result in decreased power requirements but would also improve the transfer characteristics of the device. As stated earlier, $\mathrm{ChG}$ exhibits strong normal dispersion in the $1.55-\mu \mathrm{m}$ telecommunication window, many times higher than in silica. The increased effect of dispersion in longer waveguides would smoothen out the oscillatory SPM spectral features and linearize the frequency chirp of the output pulses, resulting in better transfer curve characteristics. It has been shown that the normal dispersion of $\mathrm{ChG}$ is perfectly suited to SPM-based regeneration [41].

Finally, as we mentioned, the filter design is crucial to the optimal operation of this SPM based regenerator. In particular, operating a Bragg grating-based filter in transmission requires a good strategy to control the grating shape, width, and dispersion. Using chirped gratings can go some way to achieving these goals, including 1) wider bandwidths resulting in reduced out of band leakage; 2) Gaussian shaped pass band produced by the less abrupt sidewalls - a result of the effectively weaker local grating; and 3) reduced edge dispersion due to the absence of very strong, abrupt changes in the grating spectrum.

Alternatively, these devices can also incorporate Bragg gratings operated in reflection by possibly incorporating a Y-junction, with the grating located in the common arm, or using a grating assisted mode coupler to minimize insertion loss. A Gaussian shaped pass-band filter with a FWHM bandwidth of $2 \mathrm{~nm}$ based on a grating operating in reflection can be achieved through a short grating, on the order of $0.3-0.4 \mathrm{~mm}$, and results in near-optimal pulse shape, as shown in Fig. 15(d).

The combination of pulse propagation through a nonlinear waveguide and optical filtering is a common theme in optical signal processing devices such as optical time demultiplexers, wavelength converters, and all optical switches, and all of these devices are potential candidates for integration using the platform presented here. 


\section{CONCLUSION}

In conclusion, we demonstrated an integrated, all-optical signal regenerator capable of operating with subpicosecond pulses, based on Kerr nonlinearities in highly nonlinear chalcogenide glass waveguides. It is based on a combination of nonlinear self-phase modulation-induced spectral broadening followed by spectral filtering, and consists of a low loss $\mathrm{As}_{2} \mathrm{~S}_{3}$ rib waveguide followed by integrated high quality waveguide Bragg gratings. We investigated devices based on single reflection Bragg gratings as well as devices with integrated multigrating band pass filters. We demonstrated nonlinear power transfer curves for 1.4-ps optical pulses at $1550 \mathrm{~nm}$, for peak optical powers of $\sim 50 \mathrm{~W}$. We showed that the incorporation of a band pass filter over a single reflection band Bragg grating significantly improves the power transfer function and achieves good agreement with theory. Finally, we discussed future roadmaps in order to realize devices operating at subwatt peak powers and with subpicosecond pulses. Further future dimensions to integration would be possible in chalcogenide glass-based devices through the incorporation of rare earth-based amplifiers and sources - an area that has attracted significant attention.

\section{REFERENCES}

[1] N. Yamada, S. Nogiwa, and H. Ohta, "640-Gb/s OTDM signal measurement with high-resolution optical sampling system using wavelengthtunable soliton pulses," IEEE Photon. Technol. Lett., vol. 16, no. 4, pp. 1125-1127, Apr. 2004.

[2] O. Leclerc, B. Lavigne, E. Balmefrezol, P. Brindel, L. Pierre, D. Rouvillain, and F. Seguineau, "Optical regeneration at $40 \mathrm{~Gb} / \mathrm{s}$ and beyond," J. Lightw. Technol., vol. 21, no. 11, pp. 2779-2790, Nov. 2003.

[3] Z. J. Huang, A. Gray, I. Khrushchev, and I. Bennion, "10-Gb/s transmission over $100 \mathrm{~mm}$ of standard fiber using $2 \mathrm{R}$ regeneration in an optical loop mirror," IEEE Photon. Technol. Lett., vol. 16, no. 11, pp. 2526-2528, Nov. 2004.

[4] G. Raybon, Y. Su, J. Leuthold, R.-J. Essiambre, T. Her, C. Joergensen, P. Steinvurzel, and K. D. K. Feder, " $40 \mathrm{Gbit} / \mathrm{s}$ pseudo-linear transmission over one million kilometers," presented at the Opt. Fiber Commun. Conf., OFC 2002, Anaheim, CA, 2002.

[5] F. Ohman, S. Bischoff, B. Tromborg, and J. Mork, "Semiconductor devices for all-optical regeneration," presented at the 2003 Int. Conf. Transparent Opt. Netw., Warsaw, Poland, 2003.

[6] R. E. Slusher, G. Lenz, J. Hodelin, J. Sanghera, L. B. Shaw, and I D. Aggarwal, "Large Raman gain and nonlinear phase shifts in highpurity $\mathrm{As}_{2} \mathrm{Se}_{3}$ chalcogenide fibers," J. Opt. Soc. Amer. B, Opt. Phys., vol. 21, pp. 1146-1155, 2004.

[7] M. Asobe, T. Kanamori, and K. Kubodera, "Applications of highly nonlinear chalcogenide glass-fibers in ultrafast all-optical switches," IEEE J. Quantum Electron., vol. 29, no. 8, pp. 2325-2333, Aug. 1993.

[8] A. Zakery and S. R. Elliott, "Optical properties and applications of chalcogenide glasses: A review," J. Non-Cryst. Solids, vol. 330, pp. 1-12, 2003.

[9] S. Ramachandran and S. G. Bishop, "Photoinduced integrated-optic devices in rapid thermally annealed chalcogenide glasses," IEEE $J$. Sel. Topics Quantum Electron., vol. 11, no. 1, pp. 260-270, Jan.-Feb. 2005.

[10] R. G. DeCorby, N. Ponnampalam, M. M. Pai, H. T. Nguyen, P. K. Dwivedi, T. J. Clement, C. J. Haugen, J. N. McMullin, and S. O. Kasap, "High index contrast waveguides in chalcogenide glass and polymer," IEEE $J$. Sel. Topics Quantum Electron., vol. 11, no. 2, pp. 539-546, Mar.-Apr. 2005.

[11] S. Ramachandran and S. G. Bishop, "Low loss photoinduced waveguides in rapid thermally annealed films of chalcogenide glasses," Appl. Phys. Lett., vol. 74, pp. 13-15, 1999.

[12] A. V. Rode, A. Zakery, M. Samoc, R. B. Charters, E. G. Gamaly, and B. Luther-Davies, "Laser-deposited $\mathrm{As}_{2} \mathrm{~S}_{3}$ chalcogenide films for waveguide applications," Appl. Surf. Sci., vol. 197, pp. 481-485, 2002.
[13] A. Zakery, "Low loss waveguides in pulsed laser deposited arsenic sulfide chalcogenide films," J. Phys. D, Appl. Phys., vol. 35, pp. 2909-2913, 2002.

[14] A. Zoubir, M. Richardson, C. Rivero, A. Schulte, C. Lopez, K. Richardson, N. Ho, and R. Vallee, "Direct femtosecond laser writing of waveguides in $\mathrm{As}_{2} \mathrm{~S}_{3}$ thin films," Opt. Lett., vol. 29, pp. 748-750, 2004.

[15] J. F. Viens, C. Meneghini, A. Villeneuve, T. V. Galstian, E. J. Knystautas, M. A. Duguay, K. A. Richardson, and T. Cardinal, "Fabrication and characterization of integrated optical waveguides in sulfide chalcogenide glasses," J. Lightw. Technol., vol. 17, no. 7, pp. 1184-1191, Jul. 1999.

[16] K. Tanaka, N. Toyosawa, and H. Hisakuni, "Photoinduced Bragg gratings in $\mathrm{As}_{2} \mathrm{~S}_{3}$ optical fibers," Opt. Lett., vol. 20, pp. 1976-1978, 1995.

[17] M. Asobe, T. Ohara, I. Yokohama, and T. Kaino, "Fabrication of Bragg grating in chalcogenide glass fibre using the transverse holographic method," Electron. Lett., vol. 32, no. 17, pp. 1611-1613, Aug. 1996.

[18] A. Saliminia, K. Le Foulgoc, A. Villeneuve, and T. Galstian, "Photoinduced Bragg reflectors in As-S-Se/As-S based chalcogenide glass multilayer channel waveguides," Fiber Integr. Opt., vol. 20, pp. 151-158, 2001.

[19] S. Ramachandran, S. G. Bishop, J. P. Guo, and D. J. Brady, "Fabrication of holographic gratings in $\mathrm{As}_{2} \mathrm{~S}_{3}$ glass by photoexpansion and photodarkening," IEEE Photon. Technol. Lett., vol. 8, no. 8, pp. 10411043, Aug. 1996.

[20] T. G. Robinson, R. G. DeCorby, J. N. McMullin, C. J. Haugen, S. O. Kasap, and D. Tonchev, "Strong Bragg gratings photoinduced by 633-nm illumination in evaporated $\operatorname{AS}(2) \operatorname{Se}(3)$ thin films," Opt. Lett., vol. 28, pp. 459461, 2003.

[21] N. Ponnampalam, R. G. DeCorby, H. T. Nguyen, P. K. Dwivedi, C. J. Haugen, J. N. McMullin, and S. O. Kasap, "Small core rib waveguides with embedded gratings in $\mathrm{As}_{2} \mathrm{Se}_{3}$ glass," Opt. Express, vol. 12, pp. 6270-6277, 2004.

[22] R. Vallee, S. Frederick, K. Asatryan, M. Fischer, and T. Galstian, "Realtime observation of Bragg grating formation in $\mathrm{As}_{2} \mathrm{~S}_{3}$ chalcogenide ridge waveguides," Opt. Commun., vol. 230, pp. 301-307, 2004.

[23] Y. L. Ruan, W. T. Li, R. Jarvis, N. Madsen, A. Rode, and B. Luther-Davies, "Fabrication and characterization of low loss rib chalcogenide waveguides made by dry etching," Opt. Express, vol. 12, pp. 5140-5145, 2004.

[24] D. Freeman, S. Madden, and B. Luther-Davies, "Fabrication of planar photonic crystals in a chalcogenide glass using a focused ion beam," Opt. Express, vol. 13, pp. 3079-3086, 2005.

[25] M. Asobe, "Nonlinear optical properties of chalcogenide glass fibers and their application to all-optical switching," Opt. Fiber Technol., vol. 3, pp. 142-148, 1997.

[26] G. Lenz, J. Zimmermann, T. Katsufuji, M. E. Lines, H. Y. Hwang, S. Spalter, R. E. Slusher, S. W. Cheong, J. S. Sanghera, and I. D. Aggarwal, "Large Kerr effect in bulk Se-based chalcogenide glasses," Opt. Lett., vol. 25, pp. 254-256, 2000.

[27] P. V. Mamyshev, "All-optical data regeneration based on self-phase modulation effect," presented at the Eur. Conf. Optic. Commun., Madrid, Spain, 1998.

[28] M. Shokooh-Saremi, V. G. Ta'eed, I. C. M. Littler, D. J. Moss, B. J. Eggleton, Y. Ruan, and B. Luther-Davies, "Ultra-strong, well-apodised Bragg gratings in chalcogenide rib waveguides," Electron. Lett., vol. 41, no. 13 , pp. 738-739, Jun. 2005.

[29] J. Mork, F. Ohman, and S. Bischoff, "Analytical expression for the bit error rate of cascaded all-optical regenerators," IEEE Photon. Technol. Lett., vol. 15, no. 10, pp. 1479-1481, Oct. 2003.

[30] M. Rochette, J. N. Kutz, J. L. Blows, D. Moss, J. T. Mok, and B. J. Eggleton, "Bit-error-ratio improvement with $2 \mathrm{R}$ optical regenerators," IEEE Photon. Technol. Lett., vol. 17, no. 4, pp. 908-910, Apr. 2005.

[31] M. Rochette, J. L. Blows, and B. Eggleton, "An all-optical regenerator that discriminates noise from signal," presented at the 31st Eur. Conf. Opt. Commun. (ECOC), Glasgow, U.K., 2005.

[32] R. A. Soref, J. Schmidtchen, and K. Petermann, "Large Single-Mode Rib Wave-Guides in GeSi-Si and Si-on-SiO 2 ," IEEE J. Quantum Electron., vol. 27, no. 8, pp. 1971-1974, Aug. 1991.

[33] V. G. Ta'eed, D. J. Moss, B. J. Eggleton, D. Freeman, S. Madden, M. Samoc, B. Luther-Davies, S. Janz, and D. X. Xu, "Higher order mode conversion via focused ion beam milled Bragg gratings in silicon-oninsulator waveguides," Opt. Express, vol. 12, pp. 5274-5284, 2004.

[34] H. G. Frohlich and R. Kashyap, "Two methods of apodisation of fibreBragg-gratings," Opt. Commun., vol. 157, pp. 273-281, 1998.

[35] P. Yeh, Optical Waves in Layered Media. New York: Wiley, 1988.

[36] I. N. Duling, "All-fiber ring soliton laser mode-locked with a nonlinear mirror,” Opt. Lett., vol. 16, pp. 539-541, 1991. 
[37] N. Ho, J. M. Laniel, R. Vallee, and A. Villeneuve, "Photosensitivity of $\mathrm{As}_{2} \mathrm{~S}_{3}$ chalcogenide thin films at $1.5 \mathrm{mu}$ m," Opt. Lett., vol. 28, pp. 965967, 2003.

[38] G. P. Agrawal, Nonlinear Fiber Optics. San Diego, CA: Academic, 1989

[39] G. Lenz, B. J. Eggleton, C. R. Giles, C. K. Madsen, and R. E. Slusher, "Dispersive properties of optical filters for WDM systems," IEEE J. Quantum Electron., vol. 34, no. 8, pp. 1390-1402, Aug. 1998.

[40] I. C. M. Littler, M. Rochette, and B. J. Eggleton, "Adjustable bandwidth dispersionless bandpass FBG optical filter," Opt. Express, vol. 13, pp. 3397-3407, 2005 .

[41] L. B. Fu, M. Rochette, V. G. Ta'eed, D. J. Moss, and B. J. Eggleton, "Investigation of self-phase modulation based optical regeneration in single mode $\mathrm{As}_{2} \mathrm{Se}_{3}$ chalcogenide glass fiber," Opt. Express, vol. 13, pp. 76377644,2005

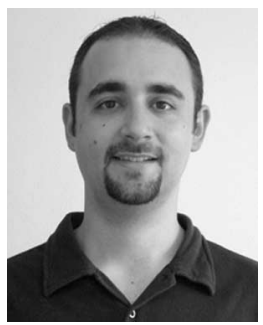

Vahid G. Ta'eed (S'05) received the B.Tech. degree in optoelectronics with first class honors from Macquarie University, Sydney, Australia, in 1998. He is currently working toward the Ph.D. degree at the Centre for Ultrahigh-bandwidth Devices for Optical Systems (CUDOS), School of Physics, University of Sydney, Sydney.

He has authored over 12 journal publications and 14 conference papers on topics ranging from integrated devices, nonlinear signal processing, waveguide Bragg gratings, high nonlinear chalcogenide glasses, fiber tapering, and photonics incorporating micro-fluidics.

Mr. Ta'eed is a member of the Optical Society of America (OSA) and is the President of the University of Sydney OSA Student Chapter.

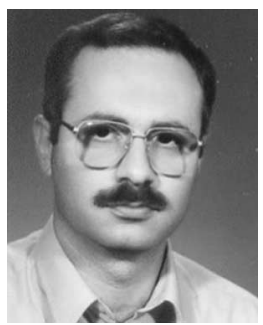

Mehrdad Shokooh-Saremi was born in Mashhad, Iran, in 1971. He received the B.S., M.S., and Ph.D. degrees from Ferdowsi University of Mashhad, Mashhad, in 1994, 1999, and 2006, respectively, all in electrical engineering.

During 2004-2005, he was with the Centre for Ultrahigh-bandwidth Devices for Optical Systems, University of Sydney, Sydney, Australia, working on fabrication and characterization of Bragg gratings and conducting nonlinear experiments in chalcogenide glasses. He is currently an Assistant Professor with the Faculty of Electrical Engineering and Robotics, Shahrood University of Technology, Shahrood, Iran. His research interests include optical periodic structures in linear and nonlinear waveguides, thin film filters, and devices based on transparent conducting oxides.

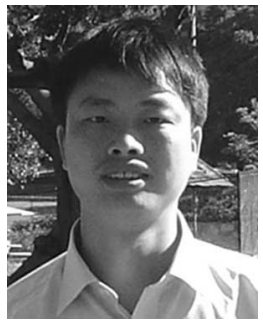

Libin Fu received the B.Sc. degree in physics from Wuhan University, Wuhan, China, in 1993, and the Ph.D. degree in optics from Peking University, Beijing, China, in 2000.

From 2000 to 2003, he was a Postdoctoral Research Fellow with the Fiber Grating Group, Optolectronics Research Center, University of Southampton,working primarily on novel fiber DFB laser design and high power double cladding fiber lasers. He is currently a Senior Research Associate at the University of Sydney, Sydney, Australia, and the Centre for Ultrahigh-bandwidth Devices for Optical Systems (CUDOS), working on all-optical signal processing and integrated nonlinear photonic circuits. His research interests include WDM devices, fiber lasers, planar waveguides, short pulse generation and amplification, and micro-structured photonic devices for high-speed communication systems.

Ian C. M. Littler, photograph and biography not available at the time of publication.

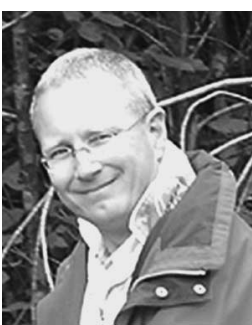

David J. Moss ( ${ }^{\prime} 83-\mathrm{M}^{\prime} 88$ ) received the B.Sc. degree in physics from the University of Waterloo, Waterloo, ON, Canada, in 1981, and the M.Sc. and Ph.D. degrees in nonlinear optics from the University of Toronto, Toronto, ON, Canada, in 1983 and 1988, respectively.

From 1988 to 1992, he was a Researcher with the National Research Council of Canada at the Institute for Microstructural Sciences, Ottawa, ON, Canada, working with the solid state optoelectronics consortium on III-V optoelectronic devices. From 1992 to 1994, he was a Senior Visiting Scientist at the Hitachi Central Research Laboratories, Tokyo, Japan, working on high-speed III-V modulators and detectors, as well as fundamental studies of quantum well tunnelling. From 1994 to 1998, he was a Senior Research Fellow at the Optical Fiber Technology Centre, University of Sydney, Sydney, Australia, working mainly in the area of silica planar waveguide devices. From 1998 to 2002, he was a Senior Scientist with JDS Uniphase, Ottawa, working in a number of areas such as fiber Bragg gratings and tunable dispersion compensation devices. He is currently a Principal Research Fellow at the University of Sydney and the Centre for Ultrahigh-bandwidth Devices for Optical Systems (CUDOS), working on all optical signal processing, integrated nonlinear photonic circuits and photonic crystal devices. He has more than 160 combined journal and conference papers.

Dr. Moss is a member of the Optical Society of America.

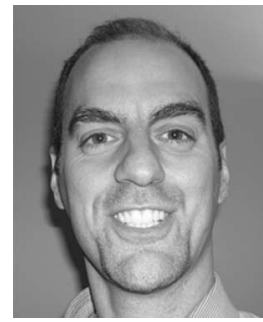

Martin Rochette received the B.S. degree in engineering physics and the M.Sc. and Ph.D. degrees in electrical and computer engineering from the Laval University, Québec City, QC, Canada, in 1995, 1997, and 2001, respectively.

In 2000 , he joined the optical communication industry where he acted as a technology leader for the modeling and the design of optical components and access systems. In 2003, he joined the Department of Electrical and Computer Engineering, Laval University, where he worked on the modeling and experiments leading to the bit error ratio of optical code division multiple access (OCDMA) systems that include incoherent and coherent optical sources. Since 2004, he has been a Research Fellow at the Centre for Ultrahigh-bandwidth Devices for Optical Systems (CUDOS), University of Sydney, Sydney, Australia where he conducts research on optical regeneration and optical monitoring for high data rate communication systems. His other research interests include nonlinear effects, all-optical processing, and high pulse rate sources. 


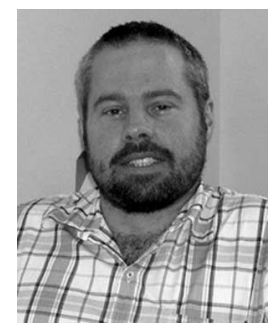

Benjamin J. Eggleton received the B.Sc. (honors) degree and the Ph.D. degree in physics from the University of Sydney, Sydney, Australia, in 1992 and 1996, respectively.

In 1996, he joined Bell Laboratories, Lucent Technologies, as a Postdoctoral Member of staff and then transferred to the Optical Fiber Research Department. In 2000, he was promoted to Research Director within the Specialty Fiber Business Division, where he was responsible for forward-looking research supporting Lucent Technologies business in optical fiber devices.

$\mathrm{He}$ is currently an ARC Federation Fellow and a Professor of Physics at the University of Sydney and the Research Director of the Centre for Ultrahighbandwidth Devices for Optical Systems (CUDOS), an ARC Centre of Excellence. He has coauthored over 140 journal publications and numerous conference papers.

Prof. Eggleton is a Fellow of the Optical Society of America (OSA). He was the recipient of the 2004 Prime Ministers Malcolm McIntosh Science Prize for Physical Scientist of the year, the 2003 ICO prize from the International Commission on Optics, the 1998 Adolph Lomb Medal from the OSA, and the Distinguished Lecturer Award from the IEEE/LEOS. He is also the recipient of an R\&D100 Award.

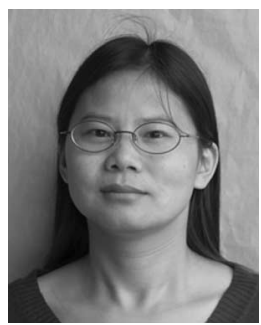

Yinlan Ruan received the B.S. and M.S. degrees from Huazhong University of Science and Technology, Wuhan, China, in 1991 and 1996, respectively, and the Ph.D. degree from the Australian National University, Canberra, Australia, in 2006.

She joined Accelink Technologies as a photonics product developer in 1996. In 2005, she joined the Centre of Expertise in Photonics, University of Adelaide, Adelaide, Australia, as a Postdoctoral Fellow. She has authored or coauthored over ten journal publications and 14 conference papers. Her research interests include special glasses for mid-infrared applications, high nonlinear materials, microstructured fibers, environmental and biological sensors, planar nonlinear waveguide, and photonics crystal devices for all-optical processing.

Dr. Ruan is a member of the Optical Society of America. She was the recipient of the 2005 Endeavour Australia Cheung Kong Award and the 2005 Chinese government award for outstanding self-financed students abroad.

Barry Luther-Davies (M'93-SM'95) photograph and biography not available at the time of publication. 